Beihefte zur Zeitschrift für die alttestamentliche Wissenschaft

Herausgegeben von

John Barton - Reinhard G. Kratz

Choon-Leong Seow $\cdot$ Markus Witte

Band 374

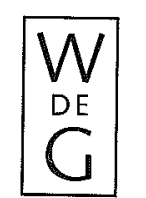

Walter de Gruyter · Berlin · New York
Studien zu Ritual und Sozialgeschichte im Alten Orient /

Studies on Ritual and Society in the Ancient Near East

Tartuer Symposien 1998-2004

\section{Herausgegeben von / Edited by}

Thomas Richard Kämmerer

\section{3}

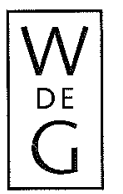

Walter de Gruyter · Berlin · New York 
1. Eröffnungssymposium: „Zur Einführung in die Altorientalistik“ (Herbst 1998),

2. Symposium: „Mesopotamien und dessen Beziehungen zu den Nachbarregionen“" (26. April - 28. April 1999),

Inhalt

3. Symposium: „Gesellschaftliche Beziehungen im Alten Vorderen Orient" (24. April - 27. April 2000).

Das für das Jahr 2001 geplante Symposium wurde mit dem internationalen Assyriologenkongress (RAI), der gemeinsam von den Universitäten Helsinki (Finnland) und Tartu (Estland) organisiert wurde, zusammengelegt.

4. Symposium: „Kunst und Magie im Alten Vorderen Orient" (22. April 25. April 2002),

5. Symposium: „Zur Geschichte der Naturwissenschaften des Alten Vorderen Orients: Sektion Medizingeschichte“ (28. April - 01. Mai 2003),

6. Symposium: „Magie und Religion“ (26. April - 28. April 2004),

7. Symposium: „Mesopotamien und die Mittelmeerregionen“ (25. April 27. April 2005),

8. Symposium: „Zur Geschichte des Transfers von Techniken im Mittelmeerraum"c (22. Mai - 24. Mai 2006),

9. Symposium: „Ideologien im Antiken Ost-Mittelmeerraum: vergleichende Annäherungen" (04. Juni - 07. Juni 2007).

Auf Grund des Exfolges und der neuen, interdisziplinären Ausrichtung des neunten und der zukünftigen Symposien, bedingt durch die Gründung des „Baltic Branchs of the Fondazione Mediterraneo (BBFM)“ an der Universität Tartu werden die Publikationen des „International Congress for East-Mediterranean Studies (ICAEM)" unter dem Zeitschriftennamen „Acta Mediterranea Tartuensia" (AcMT) erscheinen.

Vorwor

Einleitung

The Soul's Ascent and Tauroctony: On Babylonian Sediment

in the Syncretic Religious Doctrines of Late Antiquity

Amar.Annus, Tartu

Ugarit und seine Beziehungen zu Zypern und zur ägäischen Inselwelt

Manfried Dietrich, Münster

Enki / Ea und El - Die Götter der Künste und Magie

Manfried Dietrich, Münster

The Galgūla Family in South Judah and the Local Sanctuaries

Micbael Heltzer, Haifa

Infektionskrankheiten: ihre keilschriftliche Überlieferung

und molekularbiologische Bewertung

Thomas R. Kämmerer, Tartu

Arthur Vööbus - ein Forscher des christlichen Orients

Kalle Kasemaa, Tartu

Cimmerians in the Western Anatolia: A Chronological Note

Mait Köiv, Tartu

The Social Meaning of Greek Symposium

Anne Lill, Tartu

The Babylonian Almanac in the West

Alasdair Livingstone, Birmingham 
„Der Ursprung des größten Leids für die Juden“ (Josephus Ant. 19.366) Ronald Mayer-Opificius, Münster

Die Platane von Gortyna

Helmut Naumann, Münster

Beobachtungen zur alttestamentlichen Weisheitsliteratur

auf Grund der poetologischen Analyse (Kolometrie)

Urmas Nõmmik, Tartu

Die Hiobdichtung - ein überregionaler Dialog?

Am Beispiel der drei Freunde Hiobs

Urmas Nõmmik, Tartu

The Neo-Assyrian Ruling Class

Simo Parpola, Helsinki

Krankheit - ein Makel an heiliger Vollkommenheit.

Das Urteil altisraelitischer Priester in Leviticus 13 in seinem Kontext

Henning Graf Reventlow, Bocbum

Rituelle Überlieferungen in Lev 1-4 als kanaanäisches Erbe

Henning Graf Reventlow, Bocbum

Einiges zu den altmesopotamischen Beschwörungstexten in sumerischer Sprache, besonders zu einer ungewöhnlich formulierten Beschwörung gegen die Folgen von Schlangen- und Hundebiss sowie Skorpionenstich

W.H.Ph. Römer, Baarn

Magier im Neuen Testament

Peeter Roosimaa, Tartu

Vergöttlichung der Könige von Akkade

Vladimir Sazonov, Tartu

Sozialgeschichtliche Forschung am AT und ihr theologischer Ertrag

Gottfried Sprondel $\%$ Osnabrïck
Beobachtungen zur Entwicklung des Korpus lexikalischer Texte in Assur

Frauke Weiershäuser, Göttingen

Indices 


\title{
Beobachtungen zur Entwicklung des Korpus lexikalischer Texte in Assur
}

\author{
Frauke Weiershäuser, Göttingen
}

Bereits unter den ersten keilschriftlichen Texten, die in der zweiten Hälfte des vierten Jahrtausends in Mesopotamien niedergeschrieben wurden, finden sich verschiedene Wort- und Gegenstandslisten. Im Verlauf des zweiten Jahrtausends entwickelte sich ein umfangreiches Korpus verschiedener lexikalischer T'exte, deren Inhalt von einfachen Silben- und Zeichenlisten für den Schulunterricht bis zu komplexen thematischen oder etymologischen Listen reichte.

In der zweiten Hälfte des zweiten Jahttausends finden sich lexikalische Texte im gesamten Verbreitungsgebiet der Keilschrift, nicht nur im mesopotamischen Kernland Assyrien und Babylonien, sondern an vielen Orten, an denen Keilschrifttexte in größerem Umfang zu Tage traten, darunter in Hattuša, Alalah, Ugarit, Emar und Tell el-Amarna. Es fragt sich nun, auf welchen Wegen diese Texte verbreitet, den jeweiligen lokalen Bedürfnissen angepasst und erweitert wurden, bis sie im Laufe der ersten Hälfte des ersten Jahrtausends v. Chr. in einer standardisierten Fassung ${ }^{1}$ in ganz Mesopotamien vereinheitlicht worden waren. Welche Unterschiede lassen sich nun zwischen den Überlieferungen einzelner lexikalischer Serien im Kernland und in der Peripherie in der mittelassvrischen Zeit beobachten? Wann, wo und unter welchem Einfluss wurden die standardisieren Fassungen erarbeitet, wie sie uns aus dem ersten Jahrtausend bekannt sind?

In diesem Artikel soll insbesondere der Frage nachgegangen werden, welche Stellung die Schreiber in Assur bei diesem Prozess einnahmen und welche Veränderungen sich in Assur selber zu Beginn der neuassyrischen Zeit beobachten lassen.

1 Zur Frage der Kanonisierung bzw Standardisierung siehe M. Civil, MSL 14, S. 168-169; F. Rochberg-Halton, Canonicity in Cuneiform Texts. JCS 36 (1984), S. 127-144; N. Veldhuis, Continuity and change in the Mesopotamian lexical tradition. In: B. Roest, H. Vanstiphout (Hg.): Aspects of Genre and Type in Pre-Modern Literay Cultures. COMES Communications 1 (Groningen 1999) S. 112. 
Die lexikalischen Texte aus Assur datieren in die Zeit zwischen dem 13. und dem 7. Jahrhundert v.Chr. Bisher sind keine altbabylonischen oder altassyrischen 'Textvertreter bekannt. Doch das Korpus lexikalischer Texte aus Assur zeigt sich seit dem Beginn der uns erhaltenen Überlieferung voll entwickelt und es ist davon auszugehen, dass derartige Texte auch vor der mittelassyrischen Zeit in Assur verwendet wurden.

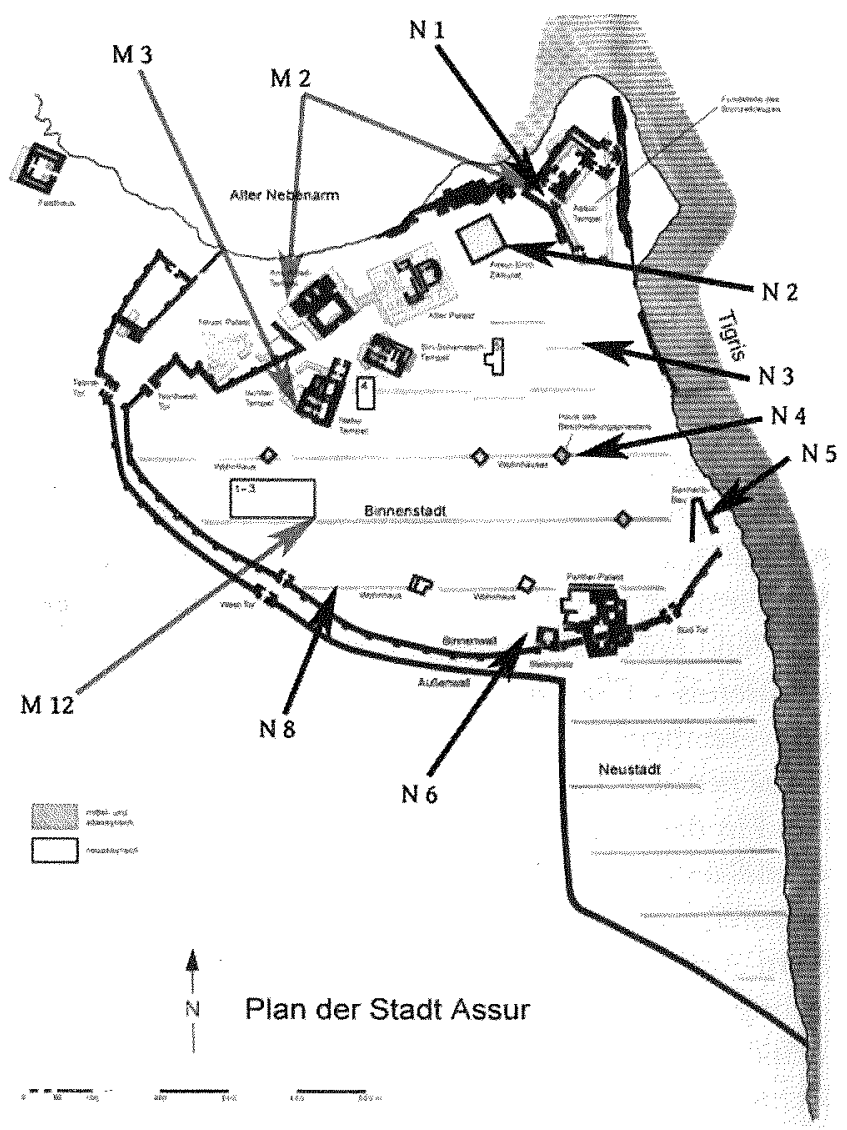

Abb. 1: Fundorte lexikalischer Texte in Assur (aus Marzahn/Salje (Hg.): Wiedererstehendes Assur. 100 Jahre Deutsche Ausgrabungen in Assyrien, Mainz 2003, Nachsatz.)
Bisher sind über 400 Texte und Fragmente lexikalischen Inhalts aus Assur bekannt, welche zu 18 verschiedenen Serien gehören. Die Tafeln stammen von unterschiedlichen Fundorten, die über das Stadtgebiet verteilt sind. Es fanden sich lexikalische Texte im Kontext offizieller Gebäude wie dem Anu-Adad-Tempel und dem Assur-Tempel e-benso wie in privaten Gebäuden. Pe-dersén hat in „Archives and Libraries in the City of Assur" die einzel-nen Tafeln nach ihren Fundkontexten zusammenge-stellt. Alle Fundorte, die Pedersén mit einer M-Nummer versah, stammen aus mittelassyrischer Zeit, Objekte aus Fundorten mit einer N-Nummer sind überwiegend neuassyrisch. In den Fundkomplexen N1/M2 fanden sich in einem spät-neuassyrisch erscheinenden archäologischen Kontext Tafeln sowohl aus mittelassyrische wie aus neuassyrischer Zeit (Abb. 1).2

Leider können nur ca. 25\% aller lexikalischen Texte aus Assur präzise einem Fundort zugeordnet werden. Für alle anderen Tafeln ist der genaue Fundkontext nicht mehr zu rekonstruieren.

Die Datierung der lexikalischen Texte stellt eine gewisse Schwierigkeit dar, da der überwiegende Teil dieser Tafeln kein Datum trägt. Wenn die einzelnen Tafeln nicht aus einem gesicherten Fundkontext stammen, muss die Datierung auf inhaltlichen und paläographischen Kriterien beruhen, doch ist dabei nur auf einige wenige Zeichen zurückzugreifen, welche häufig genug vorkommen, um Vergleiche zuzulassen, und die deutliche Unterschiede zwischen ihrer mittelassyrischen und der neuassyrischen Zeichenform aufweisen ${ }^{3}$. Allein paläographische Unterschiede reichen

2 O. Pedersen, Archives and Libraries in the City of Assur I, A Survey of the Material from the German Excavations. (Acta Univ. Upsaliensis, SSU 6, Upsala 1985) S. 31.

3 Vgl. die Gegenübetstellung det Zeichenformen unter Tiglat-Pileser I. und jener unter Assurbanipal bei E. Weidner, Die Bibliothek Tiglatpilesers I, AfO 16 (1952-53), S. 201. Allerdings ist die Zuweisung der von Weidner zusammengestellten Texte zu einer „Bibliothek" des Tiglat-Pileser I. heute nicht mehr haltbar. Es ist davon auszugehen, dass die von Weidner zusammengestellten Texte zu verschiedenen Tafelsammlungen gehötten, die im Bereich des Assur-Tempels wie des Anu-Adad-Tempels aufbewahrt wurden. Die hier besprochenen lexikalischen Texte sind um etwa 50 Jahre früher zu datieren als die Akzession Tiglat-Pilesers I. Siehe H. Freydank, Beitrïge zur mittelassyrischen Chronologie und Gescbicbte. (SGKAO 21, Berlin 1991) S. 75-78 und 94-96.

Vgl. auch die Zeichenlisten der in den letzten Jahren publizietten mittelassyrischen Textkorpora: S. Maul, Die Inscbriffen von Tall Bderi. (BBVOT 2, Berlin 1992) S. 56-62; E. CancikKirschbaum, Die mittelassyrischen Briefe aus Tall Šeh Hamad, Dür-Katlimmu. Bericbte der Ausgrabung Tall Sëh Hamad/ Dür-Katlimmu 4 (Texte 1) (Berlin 1996) S. 73-87 und K. Radnet, Das mittelassyrische Tontafelarcbiv von Giricano/Dunnu-sa-Uribi. (Subariu XIV, Brepols, Turahout 2004) S. 55-61. 
jedoch nicht aus, einen 'Text sicher zu datieren. Einige wenige Texte können über ein Kolophon datiert werden.

Dies ist der Fall bei Texten aus dem Fundkomplex N2, dem Archiv und der Bibliothek einer Schreiberfamilie. Der Name des Schreibers Summa-balāt aus der zweiten in dem Archiv überlieferten Generation, findet sich auf mehreren lexikalischen Texten ${ }^{4}$. Andere Texte aus dem Archiv dieser Familie datieren in die Jahre 687 und 683 v.Chr. Somit ist die Datierung der Texte aus dem Fundkomplex N2 in die späte neuassyrische Zeit gesichert.

Eine Gruppe von Tafeln aus dem Fundkomplex N1/M2 ist über Kolophone teilweise zu datieren. Der Text VAT 10466 (Silbenvokabular A) trägt den Namen des Eponymen Bēlu-libūr, eines Beamten aus der Regierungszeit des Tiglat-Pileser $\mathrm{I}^{5}$.

Ein weiterer Text aus dem Fundkomplex M2, VAT 9487 (Diri = watru Tafel 3), nennt den Eponymen Samnuha-ašarēed, der wohl in die Zeit des Aššrur-dān I gehört. ${ }^{6}$ Der Kolophon erwähnt neben dem Eponymen auch den Prüfer Bēl-aha-iddina. Dieser ist wahrscheinlich identisch mit einem Prüfer dieses Namens der im Kolophon zweier Texte des Mardukbalāssu-ēreš auftritt ${ }^{7}$. Aus dem Fundkomplex M2/N1 stammen die Tafeln der drei Brüder Marduk-balāssu-ēreš, Bēl-aha-iddina und Sīn-šumaiddina ${ }^{8}$, Söhne des Ninūrta-uballit-su, Schreibers des Königs, von denen Marduk-balāssu-ēreš und Bēl-aha-iddina gegenseitig ihre Tafeln überprüften. ${ }^{9}$ Die Tafeln dieser Schreiberfamilie sind über mehrere Kolophone mit

4 VAT 10262+12973+12956 (Etim-ḩuš = anantu Tafel 6), VAT 10270 (igi-du VAT 9000 Uruanna Tafel 2 und VAT $10143+12966$ an $=$ samú .

5 H. Freydank, Beiträge zur mittelassyrischen Cbronologie und Geschicbte, S. 125. C. Saporetti, Gli Eponimi medio-assiri, (BiMes 9, Malibu 1979) S. 153 und 160.

6 H. Freydank, Beiträge qur mittelassyrischen Chronologie und Geschicbte, S. 76f Anm. 205. C. Saporetti, Gli Eponimi medio-assin, S. 69.

7 VAT $9716($ Sig-alan $=$ nabnitu Tafel IV), MSL 16, S. 73, 76-92 Textzeuge B und VAT 10383 (ká-gal = abullut Tafel B), MSL 13, S. 234, 236-237 Textzeuge C.

8 VAT 10172 (e-a = nâqu Tafel 1), MSL 14, S. 173, 176-195 Textzeuge C. Für alle dre Brüder werden hier nur die Texte lexikalischen Inhalts zitiert. Zu anderen Texten der Brüder siehe die Belegstellen unter den jeweiligen Personennamen bei C. Saporetti, Onomastica Medio-Assira. (Studia Pohl 6, Rom 1970).

9 VAT 9552 (ana ittišr Tafel III), MSL 1, S. 32-50 Textzeuge B und VAT 9592 (ká-gal = abullu Tafel A), MSL 13, S. 231-233 Textzeuge A. Zur wechselseitigen Kontrolle der Texte durch die Brüder siehe H. Freydank, Beiträge ₹ur mittelassyrischen Chronologie und Geschichte, S. 95 Anm. 245.
Angabe eines Eponymen zu datieren, wobei die Namen Aššur-išmanni, Sohn des Abi-ili, Aššur-aha-iddina und Ikkaru auftreten. ${ }^{10}$ Diese Eponymen sind von Saporetti in die Zeit Tiglat-Pilesers I. gestellt worden. ${ }^{11}$ Wie Freydank gezeigt hat, ist die Wirkungszeit der drei Brüder jedoch in die Zeit des Ninurta-apil-Ekur und wohl auch in die frühen Jahre von Aššurdān I. einzuordnen. ${ }^{12}$

Die Tafel VAT 10457 (Ur5-ra = hubullu 'Tafel 7A) ist ebenfalls in der mittelassyrischen Zeichenform geschrieben, der im Kolophon erwähnte Eponym Še'i-Aššur hatte dieses Amt im Jahr 909 inne. ${ }^{13}$

Aus dem Gesagten folgt, dass Texte mit mittelassyrischer Zeichenform im lexikalischen Korpus aus Assur in die Zeit zwischen 1200 und 900 v.Chr. zu datieren sind. In einigen wenigen Fällen kann die Datierung über einen Eponymen im Kolophon näher eingegrenzt werden. Wie im folgenden zu zeigen sein wird, ist es neben einer ersten zeitlichen Eingrenzung über die Paläographie und einer genauen Datierung über die Nennung eines Eponymen mitunter möglich, eine nähere zeitliche Einordnung aufgrund der Reihenfolge der einzelnen Einträge im Vergleich zur Abfolge der Zeilen in der standardisierten Fassung des ersten Jahrtausends vorzunehmen.

\section{Standardisierung der lexikalischen Listen im 1. Jahrtausend}

In altbabylonischer Zeit waren die verschiedenen lexikalischen Serien noch nicht so strikt standardisiert wie im ersten Jahrtausend. So sind aus Nippur verschiedene Textzeugen der Serie $U_{r_{5}}$-ra $=$ hubullu bekannt, die sich alle in kleineren Details unterscheiden. ${ }^{14}$ Im ersten Jahrtausend hatten die Schreiber einen Kanon der verschiedenen umfangreichen lexikalischen Listen entwickelt, der in Assyrien wie in Babylonien in nahezu gleicher Form in Gebrauch war. Mit der Herausbildung von standardisierten Fassungen einzelner Listen hatte sich auch die Funktion dieser Textgattung verändert, lexikalische Texte wurden nun nicht mehr primär als Übung für

10 Vgl. H. Hunget, Babylonische und assyrische Kolophone. (AOAT 2, Neukitchen-Vluyn 1968) Nr. 43. Neben lexikalischen haben auch literarische Texte ein derartiges Kolophon.

11 C. Saporetti, Gli Eponimi medio-assiri, S. 151-154.

12 H. Freydank, Beiträge zur mitttelassyrischen Chronologie und Geschichte, S. 76-77 mit Anm. 205.

13 VAT 9487 (diri = watru Tafel III), MSL 15, S. 136, Textzeuge B. Der genaue Fundkontext dieser Tafel kann nicht mehr rekonstruiert werden.

14 N. Veldhuis, Continuity and Change in the Mesopotamian Lexical Tradition, S. 108-109. 
Schüler angesehen, sondern galten selber als Teil des literarischen Erbes, das zu bewahren war. ${ }^{15}$

Kleinere Abweichungen der einzelnen 'Textzeugen untereinander kamen auch in dieser Zeit noch vor. Als ein Beispiel mögen hier die letzten 27 Zeilen (Z. 382-409) der 14. Tafel von Ur5-ra = hubullu dienen. Jeweils zwei Textzeugen aus Babylon und aus Assur sind für diesen Abschnitt erhalten. ${ }^{16}$ Die Datierung der Tafeln aus Babylon basiert auf der Fundsituation, jene der Texte aus Assur auf paläographischen Kriterien, da ihr genauer Fundort nicht mehr rekonstruiert werden kann.

Assur:

A: LTBA I, 40 (VAT 11517, babylonische Schrift)

B: LTBA I, 44 (Const. 175f)

Babylon: K: LTBA I, 49 (nur die letzten 14 Zeilen erhalten) L: LTBA I, 3717

Textzeuge $A^{18}$

MIN

MIN

MIN

nam-maśsti

zer-man-du

$n i-d u l i b-b i$

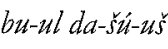

\section{MIN}

te-ni-še-e-ti

rúp-kú

$u$ u-ma-mu

MIN

「ú?-ma-mu

MIN

bu- $u-k u$

$\ulcorner$ máš 7 -anše

MIN

391 「máš ${ }^{7}-u d u$

MIN
Getier

Kleingetier

Fötus?

sechsarmig? Bez.

für Getier

Menschheit

Tiere

ditto

ditto

Vieh

ditto

ditto

15 ebd. S. 112-113. Auch im ersten Jahrtausend wurden lexikalische Texte im Schulunterricht genutzt, wie die große Zahl von Schultexten mit Exzerpten verschiedener Listen zeigt. Vgl. P. Gesche, Schulunierricht in Babylonien im ersten Jabrtausend $1 . C b r$. (AOAT 275, Münster 2000), sowie die Schultexte aus Assur VAT 8573, VAT 10071 und VAT 10756. Letztere sind kopiert bei W. G. Lambert, Babylonian Wisdom Literature (Oxford 1960) PL 73.

16 Die Texte wutden in MSL $8 / 2$ unter den Sigeln A, B, K und L bearbeitet, der fragliche Abschnitt findet sich auf den Seiten 41-43.

17 Zu LTBA I, 49 siehe O. Pedersén, Arcbive und Bibliotbeken in Babylon. Die Tontafeln der Grabung Robert Koldeweys 1899-1917. (ADOG 25, Betlin 2005), N22:4. Die Tafel stammt aus dem Bereich des Etemenanki. Zu LTBA I, 37 siehe O. Pedersén, ebd., N3:18. Die Schülertafel stammt aus dem Eingangsbereich der Südburg.

18 Die Zeilenzählung folgt hier wie in den anderen Beispielen der Zählung von MSL.

393 [níg]-「úr?-límmu-「ba` bu-lim dGìR

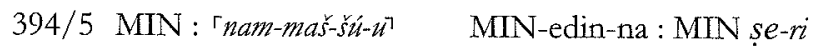

Getier des Šakkan

MIN-edin-na : MIN se-ri Wildtierherden

\begin{tabular}{|c|c|c|c|}
\hline 396 & MIN MIN & MIN dGİR & ditto des Šakkan \\
\hline 397 & níg-zi-gál & $n a m-m a \tilde{s}-\tilde{S} \mathfrak{u}-\tilde{u}$ & Getiet \\
\hline 398 & níg-zi-gál & {$[a-5 u-i u]$} & Lebewesen \\
\hline 399 & níg-zi-gál & {$\left[s i k-n a-a t n a-p i \check{s}_{-}-t e\right]$} & Lebewesen \\
\hline 400 & níg-MIN & {$[n a m-m a r-t i]$} & Getier \\
\hline 401 & níg-šu-úr & $\ulcorner\mathrm{MIN}\urcorner$ & ditto \\
\hline 402 & níg ${ }^{\text {ni-qi-ki }}$ & $\ulcorner$ ¿zir-man'-du & Kleingetier \\
\hline \multirow[t]{2}{*}{403} & níg-ki-gar & zir-man-du qaq-qar & Kleintier des \\
\hline & & & Erdbodens \\
\hline 404 & níg-gír-ùz & nam-maś-ti & Getier \\
\hline 405 & $\Gamma_{\text {anše }}{ }^{\top}-\grave{u} z$ & $\mathrm{MIN}$ & ditto \\
\hline 406 & mir-ùz & $\mathrm{MIN}$ & ditto \\
\hline 407 & mir- ${ }^{\Gamma} \mathrm{hul}^{\top}$ & bul-mit-ț $u_{4}$ & Drache \\
\hline 408 & mir- 'hul & $b u l-m a-b u$ & eine Schlange \\
\hline 409 & 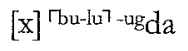 & $s e-e-r i$ & Schlange \\
\hline
\end{tabular}

Fangzeile zu Tafel 15

Die Abfolge der einzelnen Zeilen ist bei allen Textvertretern weitgehend identisch, doch zeigen sich folgende kleinere Differenzen:

- Die Zeile 384 ist nur bei Textzeuge A vorhanden.

- Die Zeilen 394/5 sind bei den Paralleltexten nicht in einer Zeile zusammengefasst.

- Textzeuge B und L haben zusätzlich eine Zeile 401a (níg-ki = MIN) eingefügt.

- Auf der Schülertafel L ist zusätzlich eine Zeile 386a (a-za-lu-lu = na-at na-pi[šsti] $)$ eingefügt.

Neben diesen Unterschieden, die Einträge ganzer Zeilen betreffen, ergeben sich zwischen den einzelnen Textvertretern Differenzen bezüglich der genauen Schreibung einzelner Einträge ${ }^{19}$.

In der Standardedition der lexikalischen Listen Mesopotamiens, der Serie „Materialien zum Sumerischen Lexikon" (MSL), werden in der Regel

19 Siehe hierzu die Anmerkungen zu den einzelnen Zeilen in MSL 8/2, S. 41-43. 
Textzeugen aus dem zweiten und dem exsten Jahrtausend aus verschiedenen Fundorten verwendet, um eine Standardversion der verschiedenen Listen zu rekonstruieren, M. Civil war der Überzeugung, die standardisierten Fassungen seien, basierend auf den altbabylonischen Vorläufern aus den großen Zentren Babyloniens, insbesondere Nippur, eventuell auch Babylon, in der kassitischen Zeit kompiliert wurden. ${ }^{20}$ Aus Babylonien sind kaum Textzeugen aus dem späten zweiten und dem frühen ersten Jahrtausend erhalten, sodass die Annahme von M. Civil für Babylonien derzeit noch nicht näher verifiziert werden kann. Allerdings zeigt sich bei genauerer Betrachtung, dass die mittelassyrischen Textzeugen aus Assur noch nicht in das Schema der standardisierten Rezensionen passen. Immer wieder finden sich in mittelassyrischen Textvertretern Zeilen, die in späteren Rezensionen nicht mehr aufgenommen worden sind. Auch die Reihenfolge der einzelnen Einträge kann in dieser Zeit noch deutlich von jenen der späteren Zeit abweichen. Mitunter sind mittelassyrische Rezensionen jedoch auch über längere Passagen vollkommen parallel zu der späteren standardisierten Fassung.

Es stellt sich nun die Frage wann, wo und unter welchem Einfluss die standardisierten Fassungen der einzelnen lexikalischen Serien, wie sie von der neuassyrischen Zeit an in Gebrauch waren, kompiliert wurden. Das entsprechende Material aus Babylonien fehlt derzeit noch weitgehend. Dagegen sind eine Reihe von Textzeugen aus dem Norden, insbesondere aus Assur, aber auch aus Nuzi, sowie aus dem Westen, aus Alalah, Hattuša, Ugarit und Emar bekannt. ${ }^{21}$ Vergleiche von Textvertretern aus diesen Orten mit den entsprechenden Passagen aus altbabylonischer Zeit einerseits und mit der standardisierten Fassung des ersten Jahrtausends andererseits können Hinweise darauf liefern, wo und unter welchem Ein-

20 M. Civil, Lexicography. In: S. J. Lieberman (Hg.): Sumerological Studies in Honor of Thorkild Jacobsen on bis seventieth Birthday June 7, 1974, (AS 20, Chicago 1976), S. 128

21 M. Civil, Lexicography, S. 128-129. Für neuere Editionen siehe u.a. zu den Texten aus Emar D. Arnaud, Recherches au pays d'Astata. Emar VI/1-4 (Paris 1985-87), zu Texten aus Ugarit B. André-Salvini, Les Textes Lexicographiques. In: P. Bordteuil (Hg.): Une bibliotbèque au sud de la ville. Les textes de la 34e campagne (1973). (Ras Shamra-Ougarit VII, Paris 1991) S. 105-126. Det überwiegende Teil der lexikalischen Texte aus Ugarit ist bislang noch unpubliziert. Zum Textbestand des lexikalischen Korpus aus Ugatit siehe W. var Soldt, Babylonian Lexical, Religious and Literary Texts and Scribal Education at Ugarit and its implication for the alphabetic literary texts. In: Manfred Dietrich, Oswald Loretz (Hg.) Ugarit. Ein ostmediterranes Kulturzentrum im alten Orient. Ergebnisse und Perspektiven der Forschung. Band I: Ugarit und seine altorientalische Umwelt. (AI ASP 7, Münster 1995), S. 171-176. fluss die verschiedenen Rezensionen einzelner lexikalischer Serien kompiliert worden sind. Ein Vergleich der Texte aus Assur mit jenen aus Emar ist dabei von besonderem Interesse, da beide Städte in direktem Kontakt zueinander standen, wohingegen direkte Kontakte zu Ugarit eher selten bezeugt sind.22 Ob Schreiber aus der Stadt Assur selber in Emar gearbeitet haben, ist derzeit nicht festzustellen, jedoch ist der Fall des assyrischen Schreibers Kidin-Gula bekannt, der, wohl aus der Region am mittleren Euphrat oder aus dem Norden Babyloniens stammend, in Emar als Lehrer gewirkt und dort auch lexikalische Listen im Unterricht verwendet hat. ${ }^{23}$ Die Tätigkeit assyrischer Schreiber außerhalb des Kerngebietes von Assyrien ist auch für Ugarit nachgewriesen. ${ }^{24}$ Somit sind Kontakte der verschiedenen lokalen Schreiberschulen und -traditionen des mesopotamischen Kernlandes mit Gebieten im Westen nicht nur mittels Textvergleichen zu erschließen, sondern in einigen Fällen sogar für bestimmte Persönlichkeiten konkret zu belegen.

Vergleicht man nun die verschiedenen Rezensionen einzelner lexikalischer Texte des zweiten Jahrtausends untereinander sowie mit der Standardrezension des ersten Jahrtausends, lassen sich sowohl Parallelen als auch deutliche Unterschiede erkennen. Hier stellt sich die Frage, auf welchen Wegen diese 'Texte weitergegeben wurden und wann und wo welche Veränderungen vorgenommen worden sind. Weiter ist zu fragen, welche Position die Schreiber der Stadt Assur bei diesem Prozess des Kulturtransfers einnahmen. Es wäre sowohl möglich anzunehmen, Assur habe als Vermittler des Wissens Babyloniens an die Zentren des Westens gewirkt, wie es auch denkbar wäre, dass Assur seinerseits Impulse aus dem Westen erhalten und aufgenommen hätte, die später Fingang gefunden haben in die Standardrezension des ersten Jahrtausends.

Im Rahmen dieses Beitrags ist eine umfassende Behandlung dieser Frage nicht möglich, es soll aber anhand von zwei Beispielen im folgenden aufgezeigt werden, wie sich die verschiedenen Rezensionen im einzelnen unterscheiden und wo Parallelen bestehen.

22 B. Faist, Der Fermbandel des assyrischen Reicbes zarischen dem 14. und 11. Jh. v.Cbr. (AOAT 265, Münster 2001) S. 216.

23 Y. Cohen, Kidin-Gula - The foreign teacher at the Emat scribal school. RA 98 (2004) S. $89-92,94$.

24 W. van Soldt, Nahišššalmu: an Assyrian sctibe working in the Southern Palace at Ugarit. In: W. van Soldt et al. (Hg.): Veenbof Anniversary Volumne. Studies presented to Klaas R. Veenhof ob the Occasion of bis sixty-fifth Birthday (Leiden 2001) S. 429-444. 
Von altbabylonischen Vorläufern zur mittelassyrischen Rezension

Das erste Beispiel ist ein Auszug aus einer Liste von Gegenständen aus Holz, die in der Standardversion als Tafel 7A zu der Serie U $\mathrm{r}_{5}-\mathrm{ra}=$ hubullu (Zeile 6-28) gehört. Für den zitierten Abschnitt, in dem verschiedene Waf fen aufgeführt werden, sind altbabylonische Vorläufer aus Nippur und Sippar, eine mittelbabylonische Quelle aus Ur sowie mittelassyrische Textzeugen aus Assur, Emar und Ugarit erhalten. Es sind bisher keine neuas syrischen Textvertreter bekannt, weswegen in MSL die Quellen aus Ur und Assur zur Rekonstruktion der "Standardversion" herangezogen wurden. Dieses Vorgehen ist nicht unproblematisch, da die Rezensionen aus mittelassyrischer Zeit von den neuassyrischen deutlich abweichen können, wie im zweiten Beispiel zu zeigen sein wird. ${ }^{25}$

Der Textvertreter aus Ur ist in der folgenden Tabelle nicht mit aufgenommen, da er weitgehend parallel zu der Rezension aus Assur ist. Nur die altbabylonischen Texte sind einsprachig sumerisch, die Rezensionen aus Assur, Ugarit und Emar sind sumerisch-akkadisch, auf die vollständige Wiedergabe der akkadischen Einträge wurde hier verzichtet, um die Übersichtlichkeit zu wahren und aufgrund des schlechten Erhaltungszustands der akkadischen Einträge insbesondere des Textes aus Emar. Für den Text aus Assur ist nur die Zeilenzählung angegeben, die sumerischen Einträge sind weitgehend parallel zu anderen Textzeugen. Zu den Differenzen bezüglich der akkadischen Einträge siehe unten. Sind einzelne Zeilen in der Übersicht freigelassen, so sind diese nicht abgebrochen, sondern fehlen bei dem betreffenden Textvertreter.

Nippur: Ni II, 127, Veldhuis, Elementary Education at Nippur, S. 162 Z. 496-502

Sippar: Si 720, MSL 6, S. 149-150, Z. 78-83

Assur: $\quad$ LTBA I, 17, MSL 6, S. 84-86 Textzeuge A

Emat: Emar VI, 4 No. 545, S. 77 Z. $450^{\circ}-461^{\circ}$

Ugarit: RS 34.180,2, André-Salvini, Les Textes Lexicographiques, S. 107 No. 49 Z. $17-29$

$25 \mathrm{Ab}$ der Zeile 17 liegt ein neubabylonischer Paralleltext vor (F. Thureau-Dangin, Notes Assyriologiques. XIIV. Fragment de vocabulaire RA 21, S. 140-141, siehe MSL 6, S 8587, Textzeuge B), der jedoch nur bedingt parallel zu dem mittelassyrischen Text aus Assur ist, so hat dieser Text parallel zum Vertreter aus Ur die in Assur fehlenden Zeilen 18 und 20 sowie als einziger Text die Zeilen 21-22, es fehlen jedoch die in Assur vorhandenen Zeilen 28, 30 und 32-53, det Text aus Ut bricht nach Zeile $27 \mathrm{ab}$. Dieser neubabylonische Text ist in der untenstehenden Übersicht nicht aufgenommen worden.

\begin{tabular}{|c|c|c|c|c|}
\hline Nippur & Sippar & Assur & Emat & Ugarit \\
\hline gištukul & gistukul & 6 & (broken) & gištukul \\
\hline gištokul-šu & gišstukul-šu & 14 & $\min -[\quad]$ & gištukul-šu \\
\hline gišstukul-út-ra & gištukul-úr-ra & 15 & [min]-dingir-[ ] & $\begin{array}{l}\text { gišstukul-din[gir]- } \\
\text { ú[r-r]a }\end{array}$ \\
\hline gišttukul-kun & gištukul-kun & 24 & & \\
\hline gištukul-dingir & & 10 & & \\
\hline \multirow[t]{12}{*}{ gištukul-gaz } & gištukul-gaz & & & \\
\hline & gištukul-ma-nu & & & \\
\hline & gištukul-dingir-ra & 9 & [min-ding]ir-ra-vii-b[e] & gisttukul-[dingir-ra] \\
\hline & & & {$[\mathrm{x}]$ min-lugal } & gištukul- - Tugal \\
\hline & & 16 & {$[\mathrm{x}]-\min -\mathrm{d} \mathrm{Da}-\mathrm{nu}$} & gišstukul-dDa-mu \\
\hline & & & kul-min-giš-ma-nu & [gis]tukul-giš- $\left\ulcorner^{m a^{\top}-n u}\right.$ \\
\hline & & & {$[x-m i n]-s ̌ e ~$} & {$[$ gištuku] $]-s ̌ e$} \\
\hline & & & {$[\mathrm{min}]-\mathrm{min}-\mathrm{še}-\mathrm{gišs}-\mathrm{i}$} & [gišstuku]l-še-ì-gišs \\
\hline & & & [min]-min-zú-lum & 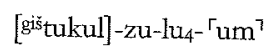 \\
\hline & & 19 & tukul-sag-n[a $\left[a_{4}\right]$ & [gištukul]-sag-na \\
\hline & & & tukul-giš & \\
\hline & & & & [gisttukul]-sag \\
\hline \multirow[t]{2}{*}{ gišsag-tukul } & gišsag-tukul & 28 & & [gìs sag-du-tuk]ul \\
\hline & & 29 & sag-tukul-sag & \\
\hline
\end{tabular}

Die beiden altbabylonischen Textzeugen weisen nur geringfügige Differenzen auf. In der Assur-Rezension sind die meisten altbabylonischen Einträge übernommen worden, jedoch in veränderter Reihenfolge. Es fehlen der Eintrag gištukul-gaz und der nur aus Sippar belegte Eintrag gištukul-ma-nu. Gegenüber den altbabylonischen Vorläufern sind in der Assurrezension die Einträge gištukul-dDa-mu und gištukul-sag-na4 aufgenommen worden. Die Rezensionen aus Emar und Ugarit folgen in der Abfolge der Zeilen enger der altbabylonischen Vorlage, jedoch fehlen die noch in Assur enthaltenen Einträge gištukul-kun und gištukul-dingir. Neben den schon in Assur hinzugefügten Einträgen nehmen die Schreiber aus Emar und Ugarit noch eine Reihe neuer Begriffe in die Listen auf. Weitere Unterschiede sind bei den erhaltenen (oben nicht wiedergegebenen) akkadischen Einträgen zu beobachten: 
- Zeile 19:

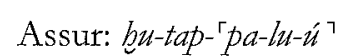

Emar: [b]u-ut-[p]á-lu-u

Ugarit: $\grave{s a-g i-i a-b a-[\quad]}$

- Während die altbabylonischen Vorläufer jeweils nur einen Eintrag gišsag-tukul aufführen, kennt der Assurtext zwei akkadische Gleichungen für diesen Eintrag26:

Zeile 28: [ŠU]- ${ }^{\ulcorner} m a^{\top}$ (= sagtukullu)

Zeile 29: $[r e-e s\}^{\top}{ }^{\top} k a k k^{\top}-k i$

In Emar wurde offensichtlich der Eintrag der Zeile 29 aufgenommen [q]a-qa-[ad ], wohingegen man in Ugarit die Gleichung der Zeile 28 listete $-\check{s} a-g a-d u-t[u[\quad]$.

Die Textvertreter aus Assur, Emar und Ugarit sind nicht genau zu datieren. Der Assurtext stammt aus dem Fundkomplex N1/M227, in dem einige mittelassyrische Texte, wie oben besprochen, über ein Kolophon auf die Regierungszeit der Könige Ninūrta-apil-Ekur (1191-79) und Assur-dan I (1178-33) zu datieren sind. Die Archive von Emar enden um das Jahr 1187, im gleichen Zeitraum wie jene von Ugarit.28

Aufgrund dieser Datenlage ist eine Beeinflussung in beiden Richtungen denkbar, also ein Einfluss assyrischer Schreiber auf die Textkompositionen in Emar und Ugarit und umgekehrt. Für ersteres könnte sprechen, dass in den Rezensionen aus Emar und Ugarit die gegenüber den altbabylonischen Fassungen in Assur hinzugefügten Zeilen enthalten sind. Auffällig ist jedoch, dass die Texte aus Emar und Ugarit bezüglich der Zeilenabfolge den altbabylonischen Texten sehr viel näher sind. Denkbar wäre auch, dass die altbabylonischen Texte über die Route am oberen Euphrat den Weg nach Westen genommen haben, und dass im 13. und 12. Jahrhundert unter gegenseitiger Beeinflussung in den verschiednen Zentren von den Schreibern unterschiedliche Rezensionen einzelner Listen entwickelt wurden. Diese wichen teilweise noch deutlich von der späteren Standardrezension ab, wie das folgende Beispiel verdeutlichen kann.

26 Die Ergänzungen folgen der Edition in MSL 6.

27 O. Pedersén, Archives and Libraries in the City of Assur II, N1:17.

$28 \mathrm{Zu}$ Emar siehe M. R: Adamthwaite, Late Hittite Emar, The Cbronology, Synchronism, and SocioPolitical Aspects of a Late Bronze Age Fortress Tom. (ANES S 8, Louvain 2001) S. XTX mit weiterer Literatur. Zu Ugarit siehe M. Yon, The City of Ugarit at Tell Ras Shamra. (Winona Lake, IN 2006), S. 21-25 mit weiterer Literatur.
Die Entwicklung der standardisierten Fassung von $\mathrm{Ur}_{5}$-ra $=$ hubullu Tafel 14

Für den folgenden Abschnitt sind neben den unten zitierten Tafeln noch weitere Textzeugen erhalten, die alle die standardisierte Fassung des ersten Jahrtausends wiedergeben. ${ }^{29}$ Für den untenstehenden Abschnitt der 14. Tafel der Serie $\mathrm{Ur}_{5}-\mathrm{ra}=$ hubullu, in dem verschiedene Wildtiere aufgeführt werden, sind wir in der glücklichen Situation, aus Assur sowohl eine mittelassyrische Rezension wie auch mehrere Exemplare der Standardrezension ethalten zu haben womit sich Parallelen und Unterschiede des gleichen Textes aus einem Fundort studieren lassen. Etwa zeitgleich mit dem mittelassyrischen Text aus Assur ${ }^{30}$ ist der Paralleltext aus Emar anzusetzen. Die Einträge dieser Rezension entsprechen weitgehend jenen der Tafeln aus Assur, weswegen hier für den Emartext nur die Zeilenzählung nach der Edition von Arnaud angegeben ist. Zu größeren Abweichungen siehe unten.

Einträge der mittelassyrischen Texte aus Assur und Emar, die nicht in det Standardrezension aufgenommen sind, werden im folgenden hervorgehoben.

Emar: $\quad$ Emar VI, 4 No. 551, S. 115 Z. 376-54“

Assur: ITBA I, 42, MSL 8/2, S. 17-18 Textzeuge J (mittelassyrisch)

Assur: LTBA I, 40, MSL 8/2, S. 16-18 Textzeuge A (neuassyrisch)

neuassyrische Standardrezension mittelassyrisch

Emar

120 az

121 ug

$a-s i$

$u_{4}-m u$

122 ug-gal

123 pirig

124 pirig

125 pirig

126 pirig-ka-dus-a

127 pirig-MIN

128 pirig-ka-tab-ba

MIN

bu-zí

lab-bi

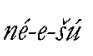

$n a-a d-r i$

${ }^{r}$ kat ${ }^{7}-t i l-i n$

129 pirig-huš

MIN

MIN

29 Dies sind nach den Sigeln von MSL 8/2 folgende Texte: B: LTBA I, 44 aus Assur; C. CT 14, 1 aus Kuyunjik; D: LTBA I, 45 aus Assur; F: LTBA I, 46 aus Assur; Schultexte S4: LTBA I, 45 aus Babylon und S7: Iraq 6, Nr. 72 aus Kiš.

30 Der genaue Fundort der Tafeln LTBA I 40 und 42 ist heute nicht mehr zu rekonstruieren. 
$\begin{array}{ll}130 \text { pirig-šu-zi-ga } & n a-a d-r i \\ 131 \text { pirig-ugu-dili } & \text { MIN }\end{array}$

neuassyrische Standardrezension mittelassvrisch Emar

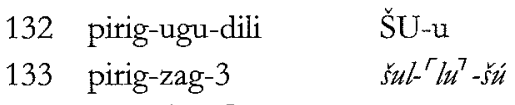

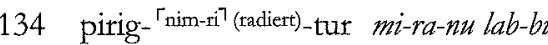

135 pirig-nim-ii_tur $\quad r_{n i}{ }^{7}$-im-ri

136 pirig-tur-bàn-da MIN $e k-d u$

tis

MIN $e k-d u \quad$ (Anfang abgebrochen)

$137 \mathrm{a}$

$137 \mathrm{~b}$

$137 \mathrm{c}$

$137 d$

138 mutum-ma

$140^{31}$ téš-bi-kú

141 téš-bi-kú

142 [udu]-idim

143 'šeg $_{9}{ }^{7}$

144 'šeg $_{9}{ }^{7}-$ bar

$144 \mathrm{a}$

145 lu-lim

142

$144 \mathrm{~b}$

$146 \mathrm{a}$

146 si-mul

$147 \mathrm{rdara}_{3}{ }^{7}$

148 daraz- ${ }^{\top}$ maš

149 daraz-maš- 'dà

$150 \mathrm{r}^{\mathrm{dara}}{ }_{3}{ }^{7}$-hyal-ḩal- ${ }^{\ulcorner} \mathrm{la}$ $a-r u-u ́ t$

$\left[\mathrm{t}_{8}\right]^{\text {mušen }} \quad<a>-r u-\dot{u}$

[ti. $]$-uš $\quad \check{\mathrm{S}} \mathrm{U}$

[tì] $]$-uš na-ad-ru

[tis-uś]-gu-la MIN

[tis-uś]-gu-la ni-i-ru

$z i-i-b i$

MIN

$a-k i-i z u$

$b i-{ }^{r} i b-b i^{7}$

$a-t u-d u$

sap-pa-ri

šinig

šinig

a-lim

lu-lim-mu lu-lim

'udu-idim?

alim $^{32}$

si-mul

$r^{7}-a-r i \quad$ si-mul

$r_{t u-r a}{ }^{7}-b u \quad$ daras

$a-a-l u \quad$ daraz-maš

$r^{n} a^{7}-a-a-h u \quad$ dara3-maš-dà

'dara3-maš ${ }^{\top}-$ hal-
151 'maš

$[s a]-r b i^{7}-[t w]$

hal- ${ }^{\ulcorner}{ }^{\top}$

Deutlich ist zu erkennen, dass nur ein geringer Teil der Zeilen 120-140 schon in der Emar-Rezension enthalten war. Nur in dem mittelassyrischen Text aus Assur sind die Zeilen 137a-d zu finden, wohingegen die Zeilen $144 \mathrm{a}$ und b sowie $146 \mathrm{a}$ auch in Emar bekannt waren. Zu der Zeile 144a ist anzumerken, dass sie auch in allen anderen Textzeugen, also sowohl in Assur wie auch in Ninive und Babylon, in der Standardrezension aufgenommen wurde, nur der hier zitierte Textzeuge A aus Assur lässt diese Zeile aus.

Die Zeilen 138-141 der Standardrezension sind offensichtlich erst spät in diese Liste aufgenommen worden, da sie weder in Emar noch in der mittelassyrischen Rezension aus Assur enthalten sind. Und Zeile 142 ist zwar in allen drei oben zitierten Versionen enthalten, wurde jedoch im ersten Jahrtausend weiter vorne in der Liste platziert als in mittelassyrischer Zeit.

Auffällig ist der Eintrag der Zeile $39^{\circ}$ aus Emar: pirig-<gal $>=p i-r i-g a ́ l-$ lu-u. Dieser Text aus Emar ist der einzige bisher bekannte Beleg für den Eintrag piriggallu in einer lexikalischen Liste. Neben dem Eintrag aus Emar ist dieses Wort bisher nur in Inschriften der neuassyrischen Könige Sargon und Sanherib bezeugt. 34

Weitere Auffälligkeiten:

- Die Zeilen 124/125 und 144a/145 sind in Emar gegenüber den Texten aus Assur jeweils in umgekehrter Reihenfolge angeordnet.

- Zeile 143: Der mittelassyrische Schreiber aus Assur schreibt als einziger irrtümlich $d u$-ú-du für atüdu. Der sumerische Eintrag in Emar lautet si Sì.

- Zeile 144: Die Mehrzahl der Texte schreibt šegg-bar = sap-pa-ri. Der Schultext aus Babylon $\left(\mathrm{S}_{4}\right)$ hat dagegen den sumerischen Eintrag zàbar, und der Schreiber aus Emar notierte sa-bar = sa-pá-ru. Hier wurde offensichtlich sa-bar = saparru „Netz“ mit šeg,-bar $=$ sappāru ,Wildbock" verwechselt ${ }^{35}$

- Zeile 144a: Alle Texte aus dem mesopotamischen Kernland haben

34 Siehe CAD P, S. 395b.

35 Siehe zu den beiden Lemmata CAD S 161 und 166a. 
den akkadischen Eintrag ditānu, wohingegen in Emar li-sáa-nu geschrieben ist.

Es zeigt sich, dass die beiden mittelassyrischen Texte aus Assur und Emar starke Ähnlichkeiten untereinander und noch deutlich Unterschiede zu der Rezension des ersten Jahrtausends aufweisen. Die verschiedenen Textzeugen für die spätere, standardisierte Fassung differieren dagegen nur in kleinen Details voneinander.

\section{Zusammenfassung}

Auf der Grundlage von nur wenigen kurzen Ausschnitten einzelner Tafeln nur einer lexikalischen Serie $\left(\mathrm{Ur}_{5}-\mathrm{ra}=h u b u l l u\right)$ sind Aussagen über die Wege gegenseitiger Beeinflussung und des Austauschs von Traditionen und Ideen kaum möglich. Es ist vorstellbar, dass die Schreiber der Stadt Assur eine Vermittlerfunktion bei der Weitergabe der lexikalischen Traditionen Babyloniens zwischen dem mesopotamischen Kernland und den Zentren im Westen eingenommen haben. Die Anfänge der Listentradition in Assur sind derzeit noch nicht bekannt, in mittelassyrischer Zeit findet sich ein vollständig entwickeltes Korpus unterschiedlichster lexikalischer Texte in Assur, das noch deutlich von der Standardrezension des ersten Jahrtausends abweichen kann. Im Vergleich der mittelassyrischen Texte zu jenen aus Ugarit und Emar zeigen sich Parallelen aber auch Unterschiede, sodass derzeit noch nicht zu sàgen ist, in welche Richtung der Austausch an Wissen in dieser Zeit im vorderen Orient erfolgte. Angesichts der 'Tatsache, dass Schreiber aus Assyrien und Babylonien in den Städten im Westen als Lehrer tätig waren, kann angenommen werden, dass auf diesem Weg auch eine Verbreitung der lexikalischen Texte stattgefunden hat. Dies bedeutet jedoch nicht, dass dieser Kulturaustausch nur in eine Richtung vonstatten ging, sondern es lassen sich, wie das zweite Textbeispiel gezeigt hat, Hinweise auf eine gegenseitige Beeinflussung finden.

Die lexikalische Tradition in Mesopotamien war in der zweiten Hälfte des zweiten Jahrtausends offenbar noch nicht so uniform, wie bisher häufig angenommen wurde. Auf der Grundlage der altbabylonischen Vorläufer wurden die verschiedenen Listen überarbeitet und konnten den jeweiligen lokalen Bedürfnissen individuell angepasst werden.

Weitere Untersuchungen anhand der Vergleiche einzelner Listen aus unterschiedlichen Zentren und Epochen werden Aufschluss über die Verbreitung des lexikalischen Korpus und dessen Ausgestaltung und Entwicklung bis zu den standardisierten Rezensionen des ersten Jahrtausends geben können. Hier sind neben den Texten aus Emar' ${ }^{36}$ insbesondere die Parallelen aus Ugarit ${ }^{37}$ und Hattuša von großem Interesse. Derartige vergleichende Studien können, so ist zu hoffen, auch tiefere Einblicke in die Rolle der Schreiber einzelner Städte bei dem Prozess der Standardisierung wie auch bezüglich des Transfers von Wissen und des Kulturaustauschs im zweiten Jahrtausend geben.
36 Vgl. M. Civil, The Texts from Meskene-Emat. AuOr 7 (1989), S. 5-25.

37 Vgl. W. van Soldt, Babylonian Lexical, Religious and Literary Texts, S. 171-176. 